\title{
Analysis of Martian Analogs Using Benchtop XRD and XRF
}

Gregory Schmidt, XRD Applications Scientist, Thermo Scientific

\author{
gregory.schmidt@thermofisher.com
}

As Martian rover missions become more detailed in their analysis functions, it is important to amass accurate data on analogous material for terrestrial applications. Furthermore, as modern labs move towards smaller footprint instrumentation, the validity of testing such samples for research quality data needs to be validated. This talk will present both XRD and XRF data collected on several Martian analog terrestrial volcanic samples using both benchtop systems. 